\title{
Evolution in models of primary care
}

B ritish Columbia has jettisoned its ambitious 2013 election promise to match everyone in the province with a family doctor. It's yet another sign that governments are beginning to recognise an evolution in the provision of primary medical care - an evolution that's supported by the College of Family Physicians of Canada.

The GP for Me program had aimed to match every $\mathrm{BC}$ resident with a family physician (FP) by the end of 2015. That didn't happen, despite the fact that $\mathrm{BC}$ has 125 FPs per 100000 population - higher than the national average of 114 . Instead of individual FPs, BC will match people with a primary care team that includes doctors as well as nurse practitioners, mental health counsellors, physiotherapists and others.

The government does not see the change as a defeat.

"We make no apologies for our aspirational goal," said Kristy Anderson, media relations manager for the Ministry of Health, in an email. Thanks to the program, 50000 additional people now have access to a family doctor or primary care clinic, she said. Dr. Christie Newton, president of the BC College of Family Physicians, said the college supported GP for $\mathrm{Me}$, but the timeline may have been too ambitious.

The change in focus is intended to create a more integrated health care system, said Anderson. "The ministry, doctors and health authorities are now working on a broader primary care strategy. This strategy includes focusing on the full range of health care professionals, working as part of a team to better meet patients' needs."

That change is the right choice, according to Dr. Garey Mazowita, past president of the College of Family Physicians of Canada, and is a reflection of the way that the profession is changing. The traditional model of family practice, with a single doctor providing comprehensive care in a variety of settings including the office, nursing home and hospital, is no longer a good fit for the young doctors graduating from medical schools today.

"There are some changing generational norms on work-life balance and satisfaction," he said. "We have graduating cohorts now who want to work in teams, and they are more dissatisfied with fee-for-service systems of pay."

Moving toward more team-based, collaborative systems of primary care is a step in the right direction. "It's encouraging," said Mazowita. It's more aligned with the direction many think the health system needs to go to accommodate the increasing burden of chronic disease and complexity that family doctors are facing, he adds.

Ontario began implementing teambased primary care in 2005. Family Health Teams, which include doctors, nurse practitioners, social workers, dietitians and others, have proven popular with both patients and physicians. But the province has only 184 such teams, with no plans to add more. The teams are proving expensive to run because some provide access to professionals not usually covered by the public system, such as psychologists. Only $25 \%$ of Ontario's family physicians practise within the Family Health Team model, although more are involved in similar organizations, such as Community Health Centres and Aboriginal Health Access Centres, that also provide access to other health care professionals. Quebec and Alberta also offer team-based family medicine.

Mazowita supports this evolution, but cautions that it is vital for the teams to continue to offer the level of care that individual doctors have always provided, including strong relationships with their patients. "It's essential that they provide comprehensive and continuous care. Those factors are the ones that are most correlated with efficient and effective health care, and with safety."

Sufficient numbers of FPs are critical to providing that care, he says. Firm numbers are hard to come by, but Mazowita estimates between $10 \%$ and $20 \%$ of people across Canada lack access to a family doctor.

Family medicine had been considered a less attractive or fashionable specialty in the past, but the college is working hard to turn that around. "There has been a resurgence of interest in family medicine residency programs," said Mazowita. "The training programs are turning out more family doctors than they ever have."

$\mathrm{BC}$, for example, doubled the number of medical school places since 2003 , and nearly tripled the number of residency positions, resulting in more than 500 additional family physicians graduating. The $\mathrm{BC}$ college would like to see half of all graduates choose family medicine as their specialty - currently, $44 \%$ do so, with the number gradually increasing, said Newton. Brian Owens, St. Stephen, NB

CMAJ 2016. DOI:10.1503/cmaj.109-5283 